\title{
Electromagnetic scattering in polarizable backgrounds
}

\author{
Olivier J. F. Martin* and Nicolas B. Piller \\ Electromagnetic Fields and Microwave Electronics Laboratory, Swiss Federal Institute of Technology, \\ ETH-Zentrum, ETZ, CH-8092 Zurich, Switzerland
}

(Received 11 February 1998)

\begin{abstract}
We develop a fully vectorial formalism for the investigation of electromagnetic scattering in polarizable backgrounds, i.e., where the scatterers are not in vacuum but situated in a medium with a dielectric permittivity different from unity. Our approach is based on the Green's tensor technique and the corresponding Green's tensors for two-dimensional (2D) and three-dimensional (3D) systems are developed. The analysis of 2D systems is not restricted to the case where transverse electric (TE) and transverse magnetic (TM) modes are decoupled, but treated in a general manner. Practical examples illustrate the application of the method: scattering by a microcavity for two dimensions and color formation in opal for three dimensions.
\end{abstract}

[S1063-651X(98)13409-8]

PACS number(s): 42.25.Bs, 42.25.Fx, 42.68.Mj, 02.60.Cb

\section{INTRODUCTION}

There are many physical situations that involve scattering in a polarizable background, i.e., where the scatterers are not in vacuum but situated in a medium with a dielectric permittivity different from unity.

Such situations arise, for example, in the investigation of scatterers distributed in an infinite system, like bubbles and dust grains in deep antarctic ice [1], inclusions in crystals [2-4], point defects in semiconductors [5], or in the study of the optical properties of colloids in solutions [6].

One also comes across scattering in polarizable backgrounds when investigating a particular subpart of a complex system. Indeed, it can often be assumed, with a large system, that the surroundings of the subpart extend to infinity. This has recently been illustrated in the study of light coupling between a local probe microscope tip and a surface [7].

The aim of this paper is to develop a general formalism able to handle such situations. Our approach is based on the volume integral equation for scattering and we will develop the corresponding Green's tensors both for two-dimensional (2D) and three-dimensional (3D) systems. Furthermore, the link between 2D and 3D geometries will be emphasized and $2 \mathrm{D}$ systems will be treated in a completely general manner.

Since we will restrict the present study to infinite homogeneous backgrounds, one should note that another class of reference systems, namely, surfaces and multilayered substrates, has been developed for the Green's tensor technique [8-10]. The material developed in the present work can also be used in conjunction with these complex reference systems; for example, a subsurface scatterer is merely a scatterer placed in a polarizable background (the substrate in that case).

The formalism is developed in Sec. II, where Green's tensors for 2D and 3D systems are derived and a practical numerical implementation discussed. This technique is then illustrated with two examples of applications in Sec. III and our work is summarized in Sec. IV.

\footnotetext{
*Electronic address: martin@ifh.ee.ethz.ch
}

\section{FORMALISM}

\section{A. Solution of the scattering problem}

Let us consider a scattering system described by a dielectric function $\varepsilon(\mathbf{r})$ embedded in an infinite homogeneous background medium $\varepsilon_{\mathrm{B}}$ [throughout the paper we assume nonmagnetic materials and an $\exp (-i \omega t)$ time dependence for the fields]. The scattering system does not need to be homogeneous; furthermore it can be composed by several distinct bodies embedded in the infinite background. When the background medium is not vacuum $\left(\varepsilon_{\mathrm{B}} \neq 1\right)$, the scatterers may have a lower permittivity $\varepsilon(\mathbf{r})$ than $\varepsilon_{\mathrm{B}}$. Finally, realistic metals can be considered by using a complex value dielectric function.

When this system is illuminated by an incident field $\mathbf{E}^{0}(\mathbf{r})$ propagating in the background medium, the total electric field (incident field plus scattered field) is a solution of the vectorial wave equation

$$
\nabla \times \nabla \times \mathbf{E}(\mathbf{r})-k_{0}^{2} \varepsilon(\mathbf{r}) \mathbf{E}(\mathbf{r})=\mathbf{0},
$$

where $k_{0}^{2}=\omega^{2} / c^{2}$ is the vacuum wave number.

Although the present formalism can easily handle anisotropic scatterers described by a tensorial dielectric function, for the sake of simplicity we limit the discussion to scalar dielectric functions. To take a dielectric tensor $\boldsymbol{\varepsilon}(\mathbf{r})$ into account, the reader should simply replace in the formulas the product $\varepsilon(\mathbf{r}) \mathbf{E}(\mathbf{r})$ with the corresponding contraction $\boldsymbol{\varepsilon}(\mathbf{r}) \cdot \mathbf{E}(\mathbf{r})$.

Introducing the dielectric contrast

$$
\Delta \varepsilon(\mathbf{r})=\varepsilon(\mathbf{r})-\varepsilon_{\mathrm{B}},
$$

we can rewrite Eq. (1) as an inhomogeneous equation,

$$
\nabla \times \nabla \times \mathbf{E}(\mathbf{r})-k_{0}^{2} \varepsilon_{\mathrm{B}} \mathbf{E}(\mathbf{r})=k_{0}^{2} \Delta \varepsilon(\mathbf{r}) \mathbf{E}(\mathbf{r}),
$$

where the incident field $\mathbf{E}^{0}(\mathbf{r})$ must be a solution of the corresponding homogeneous equation:

$$
\nabla \times \nabla \times \mathbf{E}^{0}(\mathbf{r})-k_{0}^{2} \varepsilon_{\mathrm{B}} \mathbf{E}^{0}(\mathbf{r})=\mathbf{0} .
$$


To compute the total field $\mathbf{E}(\mathbf{r})$, let us introduce Green's tensor $\mathbf{G}^{\mathrm{B}}\left(\mathbf{r}, \mathbf{r}^{\prime}\right)$ associated with the infinite background $\varepsilon_{\mathrm{B}}$. This dyadic is a solution of the vector wave equation (4) with a point source term [11]:

$$
\nabla \times \nabla \times \mathbf{G}^{\mathrm{B}}\left(\mathbf{r}, \mathbf{r}^{\prime}\right)-k_{0}^{2} \varepsilon_{\mathrm{B}} \mathbf{G}^{\mathrm{B}}\left(\mathbf{r}, \mathbf{r}^{\prime}\right)=\mathbf{1} \delta\left(\mathbf{r}-\mathbf{r}^{\prime}\right),
$$

where $\mathbf{1}$ is the unit dyad.

Introducing Eq. (5) into Eq. (3), it is a simple matter to find that the total field $\mathbf{E}(\mathbf{r})$ is given by

$$
\mathbf{E}(\mathbf{r})=\mathbf{E}^{0}(\mathbf{r})+\int_{\mathrm{V}} \mathrm{d} \mathbf{r}^{\prime} \mathbf{G}^{\mathrm{B}}\left(\mathbf{r}, \mathbf{r}^{\prime}\right) \cdot k_{0}^{2} \Delta \varepsilon\left(\mathbf{r}^{\prime}\right) \mathbf{E}\left(\mathbf{r}^{\prime}\right),
$$

where the integration runs over the entire scatterer volume $V$.

In the next subsections, where we derive Green's tensors for $2 \mathrm{D}$ and $3 \mathrm{D}$ systems, we will see that $\mathbf{G}^{\mathrm{B}}\left(\mathbf{r}, \mathbf{r}^{\prime}\right)$ diverges for $\mathbf{r}=\mathbf{r}^{\prime}$. Therefore, whenever both $\mathbf{r}$ and $\mathbf{r}^{\prime}$ are inside the scatterer volume $V$, the principal value must be taken for the integral in Eq. (6) and the singularity of the Green's tensor treated separately. This is emphasized by rewriting Eq. (6) as

$$
\begin{aligned}
\mathbf{E}(\mathbf{r})= & \mathbf{E}^{0}(\mathbf{r})+\lim _{\delta \mathrm{V} \rightarrow 0} \int_{\mathrm{V}-\delta \mathrm{V}} \mathrm{d} \mathbf{r}^{\prime} \mathbf{G}^{\mathrm{B}}\left(\mathbf{r}, \mathbf{r}^{\prime}\right) \cdot k_{0}^{2} \Delta \varepsilon\left(\mathbf{r}^{\prime}\right) \mathbf{E}\left(\mathbf{r}^{\prime}\right) \\
& -\mathbf{L} \cdot \frac{\Delta \varepsilon(\mathbf{r})}{\varepsilon_{\mathrm{B}}} \mathbf{E}(\mathbf{r}),
\end{aligned}
$$

where the infinitesimal volume $\delta V$ centered at the point $\mathbf{r}$ is used to exclude the singularity. The source dyadic $\mathbf{L}$ depends on the shape of the exclusion volume $\delta V$. Its derivation is given with much detail by Yaghjian [12]. Practical values of $\mathbf{L}$ for $2 \mathrm{D}$ and 3D systems will be given in the next subsections. Note the important factor $\varepsilon_{\mathrm{B}}$ in the $\mathbf{L}$ term of Eq. (7); this factor does not appear in the work of Yaghjian as this author only considers scattering in vacuum.

When the observation point $\mathbf{r}$ is located outside the scatterer, no singularity shows up since the integration in Eq. (6) is limited to the scatterer volume. Actually, Eq. (6) shows that the field at any point in the background is entirely determined from the field inside the scatterer. This can be used to split the calculation: in a first step only the field inside the scatterer is computed and stored; the field at any desired location in the background being then computed at a later stage.

Different approaches can be used to solve Eq. (7) numerically. We will here briefly present the discrete dipole approximation (DDA, also known as the coupled dipole approximation, CDA) since it is quite simple to implement numerically and has proven to be most useful for investigating the scattering by arbitrary shape particles [13]. An alternative numerical approach would be to use finite elements [14].

To solve Eq. (7) numerically, let us define a grid with $N$ meshes over the system. Each mesh $i$ is centered at position $\mathbf{r}_{i}$ and has a volume $V_{i}, i=1, \ldots, N$ (for 2D systems $V_{i}$ represents the area of the mesh). A regular mesh with constant volume $\mathrm{V}_{i}$ is not mandatory and a higher mesh refinement can be used where a precise knowledge of the field is required or where the dielectric contrast $\Delta \varepsilon(\mathbf{r})$ is large.
Introducing the discretized field $\mathbf{E}_{i}=\mathbf{E}\left(\mathbf{r}_{i}\right)$, the discretized dielectric contrast $\Delta \varepsilon_{i}=\Delta \varepsilon\left(\mathbf{r}_{i}\right)$ and the discretized Green's tensor $\mathbf{G}_{i, j}^{\mathrm{B}}=\mathbf{G}^{\mathrm{B}}\left(\mathbf{r}_{i}, \mathbf{r}_{j}\right)$, we can rewrite Eq. (7) as a dense system of linear equations:

$$
\begin{aligned}
\mathbf{E}_{i}= & \mathbf{E}_{i}^{0}+\sum_{j=1, j \neq i}^{N} \mathbf{G}_{i, j}^{\mathrm{B}} \cdot k_{0}^{2} \Delta \varepsilon_{j} \mathbf{E}_{j} \mathrm{~V}_{j}+\mathbf{M}_{i} \cdot k_{0}^{2} \Delta \varepsilon_{i} \mathbf{E}_{i} \\
& -\mathbf{L} \cdot \frac{\Delta \varepsilon_{i}}{\varepsilon_{\mathrm{B}}} \mathbf{E}_{i}, \quad i=1, \ldots, N
\end{aligned}
$$

with

$$
\mathbf{M}_{i}=\lim _{\delta \mathrm{V} \rightarrow 0} \int_{\mathrm{V}_{i}-\delta \mathrm{V}} \mathrm{d} \mathbf{r}^{\prime} \mathbf{G}^{\mathrm{B}}\left(\mathbf{r}_{i}, \mathbf{r}^{\prime}\right)
$$

The value of the self-term $\mathbf{M}_{i}$ is usually small compared to the other terms in Eq. (8) and several authors simply set $\mathbf{M}_{i}=\mathbf{0}$. Nevertheless, our experience shows that $\mathbf{M}_{i}$ plays a non-negligible role for the accuracy of the results [15]. The value of $\mathbf{M}_{i}$ can then be obtained by numerical quadrature of Eq. (9) on mesh $i$. Another approach consists in mapping mesh $i$ onto a mesh with a simpler shape, so that Eq. (9) can be evaluated analytically. This last approach gives extremely good results for mesh shapes close to cubic meshes in three dimensions and to square meshes in two dimensions. This covers most practical situations and the corresponding values for $\mathbf{M}_{i}$ will be given in the next two subsections.

Several numerical methods can be used to solve the system of equations (8). It is important to note that when the dielectric contrast is strong or the scatterer volume important, the condition number of the matrix associated with Eq. (8) becomes quite large, thereby requiring an extremely stable linear algebra solver. Iterative solvers such as conjugate gradients seem particularly well suited for that task [16].

To conclude this section, let us mention that an alternate form of Eq. (6) exists:

$$
\mathbf{E}(\mathbf{r})=\mathbf{E}^{0}(\mathbf{r})+\int_{\mathrm{V}} \mathrm{d} \mathbf{r}^{\prime} \mathbf{G}\left(\mathbf{r}, \mathbf{r}^{\prime}\right) \cdot k_{0}^{2} \Delta \varepsilon\left(\mathbf{r}^{\prime}\right) \mathbf{E}^{0}\left(\mathbf{r}^{\prime}\right)
$$

While Eq. (6) represents an implicit equation [the unknown field $\mathbf{E}(\mathbf{r})$ appears also under the integral], Eq. (10) gives explicitly this unknowned field from the incident field $\mathbf{E}^{0}(\mathbf{r})$. The dyadic $\mathbf{G}\left(\mathbf{r}, \mathbf{r}^{\prime}\right)$ in Eq. (10) represents Green's tensor of the complete system (homogeneous background plus scatterers) and fulfills Dyson's equation,

$$
\mathbf{G}\left(\mathbf{r}, \mathbf{r}^{\prime}\right)=\mathbf{G}^{\mathrm{B}}\left(\mathbf{r}, \mathbf{r}^{\prime}\right)+\int_{\mathrm{V}} \mathrm{d} \mathbf{r}^{\prime \prime} \mathbf{G}^{\mathrm{B}}\left(\mathbf{r}, \mathbf{r}^{\prime \prime}\right) \cdot k_{0}^{2} \Delta \varepsilon\left(\mathbf{r}^{\prime \prime}\right) \mathbf{G}\left(\mathbf{r}^{\prime \prime}, \mathbf{r}^{\prime}\right) .
$$

Equation (11) is the counterpart for Green's tensor of Eq. (6). Aside from Eq. (10), Green's tensor $\mathbf{G}\left(\mathbf{r}, \mathbf{r}^{\prime}\right)$ finds numerous applications in electromagnetics, where the response of a complex system is required [17]. It can also be applied to rather subtle situations such as the investigation of the electromagnetic interaction of fluorescent molecules with their surrounding [18] or in spectroscopy [19]. 


\section{B. 3D Green's tensor}

Green's tensor $\mathbf{G}^{\mathrm{B}}\left(\mathbf{r}, \mathbf{r}^{\prime}\right)$ for an infinite homogeneous 3D background medium $\varepsilon_{\mathrm{B}}$ is readily obtained from [20]

$$
\mathbf{G}^{\mathrm{B}}\left(\mathbf{r}, \mathbf{r}^{\prime}\right)=\left(\mathbf{1}+\frac{\nabla \nabla}{k_{\mathrm{B}}^{2}}\right) g_{3 \mathrm{D}}^{\mathrm{B}}\left(\mathbf{r}, \mathbf{r}^{\prime}\right),
$$

where

$$
g_{3 \mathrm{D}}^{\mathrm{B}}\left(\mathbf{r}, \mathbf{r}^{\prime}\right)=\frac{\exp \left(i k_{\mathrm{B}} R\right)}{4 \pi R}
$$

is the scalar Green's function associated with the background, $R=|\mathbf{R}|=\left|\mathbf{r}-\mathbf{r}^{\prime}\right|$ the relative distance between source and observation points, and $k_{\mathrm{B}}$ is the wave number in the background medium:

$$
k_{\mathrm{B}}^{2}=\frac{\omega^{2}}{c^{2}} \varepsilon_{\mathrm{B}}
$$

Introducing Eq. (13) into Eq. (12) leads to the explicit form of $G^{\mathrm{B}}\left(\mathbf{r}, \mathbf{r}^{\prime}\right)$ :

$$
\begin{aligned}
\mathbf{G}^{\mathrm{B}}\left(\mathbf{r}, \mathbf{r}^{\prime}\right)= & \left(\mathbf{1}+\frac{i k_{\mathrm{B}} R-1}{k_{\mathrm{B}}^{2} R^{2}} \mathbf{1}\right. \\
& \left.+\frac{3-3 i k_{\mathrm{B}} R-k_{\mathrm{B}}^{2} R^{2}}{k_{\mathrm{B}}^{2} R^{4}} \mathbf{R R}\right) \frac{\exp \left(i k_{\mathrm{B}} R\right)}{4 \pi R} .
\end{aligned}
$$

Since the scalar Green's function only depends on the absolute relative distance $R$, Green's tensor is reciprocal, $G^{\mathrm{B}}\left(\mathbf{r}, \mathbf{r}^{\prime}\right)=G^{\mathrm{B}}\left(\mathbf{r}^{\prime}, \mathbf{r}\right)$; furthermore, the $(3 \times 3)$ matrix that represents Green's tensor is also symmetrical:

$$
\mathbf{G}^{\mathrm{B}}\left(\mathbf{r}, \mathbf{r}^{\prime}\right)=\left(\begin{array}{ccc}
G_{x x}^{\mathrm{B}} & G_{x y}^{\mathrm{B}} & G_{x z}^{\mathrm{B}} \\
G_{x y}^{\mathrm{B}} & G_{y y}^{\mathrm{B}} & G_{y z}^{\mathrm{B}} \\
G_{x z}^{\mathrm{B}} & G_{y z}^{\mathrm{B}} & G_{z z}^{\mathrm{B}}
\end{array}\right) .
$$

Finally, it is interesting to note that $G^{\mathrm{B}}\left(\mathbf{r}, \mathbf{r}^{\prime}\right)$ contains both far-field and near-field terms. While the former have an $1 / R$ dependence, the latter have $1 / R^{2}$ and $1 / R^{3}$ dependences.

For the self-term $\mathbf{M}_{i}$, integration of Eq. (9) on a spherical mesh with effective radius $R_{i}^{\text {eff }}$,

$$
R_{i}^{\mathrm{eff}}=\left(\frac{3}{4 \pi} \mathrm{V}_{i}\right)^{1 / 3}
$$

and spherical exclusion volume $\delta V$, leads to

$$
\mathbf{M}_{i}=\frac{2}{3 k_{\mathrm{B}}^{2}}\left[\left(1-i k_{\mathrm{B}} R_{i}^{\mathrm{eff}}\right) \exp \left(i k_{\mathrm{B}} R_{i}^{\mathrm{eff}}\right)-1\right] \mathbf{1}
$$

and the corresponding source dyadic is [12]

$$
\mathbf{L}=\frac{1}{3} \mathbf{1} \text {. }
$$

Thus we have all the different terms required by the system of equations (8) for a 3D geometry.

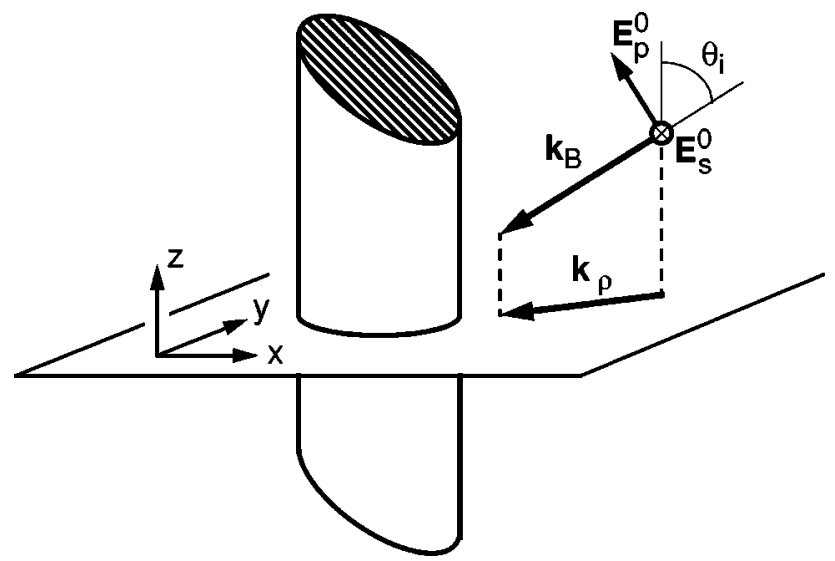

FIG. 1. Illustration of a 2D system. Although the geometry is invariant in the $z$ direction and its study can be restricted to the $x-y$ plane, the electromagnetic field needs not propagate in this plane and the incident field $\mathbf{E}^{0}$ can impinge on the system with an angle $\theta_{i} \neq 90^{\circ}$. Two different polarizations for the incident field can be considered: $p$ polarization, where the electric field is parallel to the plane of incidence and $s$ polarization, where it is orthogonal. When $\theta_{i}=90^{\circ}, p$ polarization is referred to as TM and $s$ polarization as TE.

Let us conclude this subsection with a remark on the physical signification of Green's tensor for a 3D system: $\mathbf{G}^{\mathrm{B}}\left(\mathbf{r}, \mathbf{r}^{\prime}\right)$ simply represents the electric field radiated at location $\mathbf{r}$ by three orthogonal unit dipoles (point sources) located at $\mathbf{r}^{\prime}$. More precisely, each column $\mathbf{E}_{u}$ of Green's tensor (16),

$$
\mathbf{E}_{u}=\left(\begin{array}{c}
G_{x u}^{\mathrm{B}} \\
G_{y u}^{\mathrm{B}} \\
G_{z u}^{\mathrm{B}}
\end{array}\right), \quad u=x, y, z,
$$

represents the three components of the electric field radiated by a unit dipole parallel to the $u$ axis.

\section{2D Green's tensor}

Before developing Green's tensor for a 2D system, it seems appropriate to say a few words about $2 \mathrm{D}$ geometries and their relation to $3 \mathrm{D}$ systems. A 2D geometry simply proceeds from a 3D system that exhibits a translation symmetry in one direction, like the infinite cylinder in Fig. 1. The study of such a 3D system can therefore be restricted to a plane orthogonal to the translation axis of the system $(x-y$ plane in Fig. 1).

It is, however, not necessary that the electromagnetic field also propagates in that plane and, for example, the incident field can impinge on the cylinder with a propagation vector $\mathbf{k}_{\mathrm{B}}$ nonparallel to the $x-y$ plane $\left(\theta_{\mathrm{i}} \neq 90^{\circ}\right.$, Fig. 1). In that case, all three $x, y$, and $z$ components of the electric field are coupled together and Green's tensor takes a similar form to that of Eq. (16).

On the other hand, when the field propagates in the $x-y$ plane $\left(\mathbf{k}_{\mathrm{B}}=\mathbf{k}_{\rho}, \theta_{\mathrm{i}}=90^{\circ}\right.$, Fig. 1), two distinct polarization modes can be excited: transverse electric (TE) mode, where the electric field has only two $x, y$ components and transverse magnetic (TM) mode, where the field has only one single $z$ component. In this case the elements of Green's 
tensor (16) that couple $x$ or $y$ with $z$ vanish and the two polarization modes are decoupled. Thus, when the incident field has a given polarization, the scattered field has the same polarization.

Let us define the transverse coordinate $\boldsymbol{\rho}$ :

$$
\mathbf{r}=(\boldsymbol{\rho}, z)=(x, y, z)
$$

and the transverse wave vector $\mathbf{k}_{\rho}$ :

$$
\begin{gathered}
\mathbf{k}_{\mathrm{B}}=\left(\mathbf{k}_{\rho}, k_{z}\right), \\
k_{\rho}=\left|\mathbf{k}_{\rho}\right| .
\end{gathered}
$$

As for 3D systems, Green's tensor for 2D geometries only depends on the relative position of the source and observation points; it is therefore convenient to use the relative coordinate

$$
\varrho=\boldsymbol{\rho}-\boldsymbol{\rho}^{\prime}=[\varrho \cos (\theta), \varrho \sin (\theta)]=\left(x-x^{\prime}, y-y^{\prime}\right) \text {. }
$$

Green's tensor $\mathbf{G}^{\mathrm{B}}\left(\boldsymbol{\rho}, \boldsymbol{\rho}^{\prime}\right)$ for a $2 \mathrm{D}$ system can either be developed in Fourier space [21] or deduced from the 2D Green's function,

$$
g_{2 \mathrm{D}}^{\mathrm{B}}\left(\mathbf{r}, \mathbf{r}^{\prime}\right)=\frac{i}{4} \mathrm{H}_{0}\left(k_{\rho} \varrho\right) \exp \left(i k_{z} z\right)
$$

where we use for simplicity the notation $\mathrm{H}_{i}$ for the Hankel function of the first kind $\mathrm{H}_{i}^{(1)}$ [12].

To derive the scalar Green's function (25) let us recall the physical meaning of Green's tensor: while in three dimensions it is associated with a point source, Green's tensor for a 2D system represents the field generated in an observation plane $z=$ const by an infinite line source extending in the $z$ direction with an $\exp \left(i k_{z} z\right)$ dependence. Green's function $g_{2 \mathrm{D}}^{\mathrm{B}}\left(\mathbf{r}, \mathbf{r}^{\prime}\right)$ is then readily obtained by integration over this line source:

$$
\begin{aligned}
g_{2 \mathrm{D}}^{\mathrm{B}}\left(\mathbf{r}, \mathbf{r}^{\prime}\right) & =\int_{-\infty}^{\infty} d z^{\prime} g_{3 \mathrm{D}}^{\mathrm{B}}\left(\mathbf{r}, \mathbf{r}^{\prime}\right) \exp \left(i k_{z} z^{\prime}\right) \\
& =\int_{-\infty}^{\infty} d z^{\prime} \frac{\exp \left[i k_{\mathrm{B}} \sqrt{x^{2}+y^{2}+\left(z-z^{\prime}\right)^{2}}\right]}{4 \pi \sqrt{x^{2}+y^{2}+\left(z-z^{\prime}\right)^{2}}} \exp \left(i k_{z} z^{\prime}\right) \\
& =\frac{i}{4} \mathrm{H}_{0}\left(k_{\rho} \varrho\right) \exp \left(i k_{z} z\right),
\end{aligned}
$$

where we have assumed that the line source was located at $x^{\prime}=y^{\prime}=0$. The integration of Eq. (27) is easily carried out with the help of Eq. (3.876) in Ref. [22].

Application of Eq. (12) to $g_{2 \mathrm{D}}^{\mathrm{B}}\left(\mathbf{r}, \mathbf{r}^{\prime}\right)$ as defined by Eq. (25) and evaluation of the result for $z=0$ gives Green's tensor for an infinite homogeneous $2 \mathrm{D}$ system $\varepsilon_{\mathrm{B}}$ :

$$
\mathbf{G}^{\mathrm{B}}\left(\boldsymbol{\rho}, \boldsymbol{\rho}^{\prime}\right)=\left(\begin{array}{ccc}
G_{x x}^{\mathrm{B}} & G_{x y}^{\mathrm{B}} & G_{x z}^{\mathrm{B}} \\
G_{x y}^{\mathrm{B}} & G_{y y}^{\mathrm{B}} & G_{y z}^{\mathrm{B}} \\
G_{x z}^{\mathrm{B}} & G_{y z}^{\mathrm{B}} & G_{z z}^{\mathrm{B}}
\end{array}\right),
$$

with

$$
\begin{aligned}
& G_{x x}^{\mathrm{B}}\left(\boldsymbol{\rho}, \boldsymbol{\rho}^{\prime}\right)=\frac{i}{4}\left(1-\frac{k_{\rho}^{2} \cos ^{2}(\theta)}{k_{\mathrm{B}}^{2}}\right) \mathrm{H}_{0}\left(k_{\rho} \varrho\right) \\
& +\frac{i}{4} \frac{k_{\rho} \cos (2 \theta)}{k_{\mathrm{B}}^{2} \varrho} \mathrm{H}_{1}\left(k_{\rho} \varrho\right) \text {, } \\
& G_{x y}^{\mathrm{B}}\left(\boldsymbol{\rho}, \boldsymbol{\rho}^{\prime}\right)=\frac{i}{4} \frac{k_{\rho}^{2} \sin (2 \theta)}{2 k_{\mathrm{B}}^{2}} \mathrm{H}_{2}\left(k_{\rho} \varrho\right), \\
& G_{x z}^{\mathrm{B}}\left(\boldsymbol{\rho}, \boldsymbol{\rho}^{\prime}\right)=\frac{1}{4} \frac{k_{\rho} k_{z} \cos (\theta)}{k_{\mathrm{B}}^{2}} \mathrm{H}_{1}\left(k_{\rho} \varrho\right),
\end{aligned}
$$

$$
\begin{aligned}
G_{y y}^{\mathrm{B}}\left(\boldsymbol{\rho}, \boldsymbol{\rho}^{\prime}\right)= & \frac{i}{4}\left(1-\frac{k_{\rho}^{2} \sin ^{2}(\theta)}{k_{\mathrm{B}}^{2}}\right) \mathrm{H}_{0}\left(k_{\rho} \varrho\right) \\
& -\frac{i}{4} \frac{k_{\rho} \cos (2 \theta)}{k_{\mathrm{B}}^{2} \varrho} \mathrm{H}_{1}\left(k_{\rho} \varrho\right),
\end{aligned}
$$

$$
\begin{aligned}
G_{y z}^{\mathrm{B}}\left(\boldsymbol{\rho}, \boldsymbol{\rho}^{\prime}\right) & =\frac{1}{4} \frac{k_{\rho} k_{z} \sin (\theta)}{k_{\mathrm{B}}^{2}} \mathrm{H}_{1}\left(k_{\rho} \varrho\right), \\
G_{z z}^{\mathrm{B}}\left(\boldsymbol{\rho}, \boldsymbol{\rho}^{\prime}\right) & =\frac{i}{4}\left(1-\frac{k_{z}^{2}}{k_{\mathrm{B}}^{2}}\right) \mathrm{H}_{0}\left(k_{\rho} \varrho\right) .
\end{aligned}
$$

Note that one can also replace $\mathrm{H}_{1}$ with a combination of $\mathrm{H}_{0}$ and $\mathrm{H}_{2}$, using the recurrence formulas for Hankel functions.

When the incident field propagates in the $x-y$ plane (i.e., $k_{\rho}=k_{\mathrm{B}}, k_{z}=0$, Fig. 1), Green's tensor reduces to the form

$$
\mathbf{G}^{\mathrm{B}}\left(\boldsymbol{\rho}, \boldsymbol{\rho}^{\prime}\right)=\left(\begin{array}{ccc}
G_{x x}^{\mathrm{B}} & G_{x y}^{\mathrm{B}} & 0 \\
G_{x y}^{\mathrm{B}} & G_{y y}^{\mathrm{B}} & 0 \\
0 & 0 & G_{z z}^{\mathrm{B}}
\end{array}\right),
$$

with

$$
\begin{gathered}
G_{x x}^{\mathrm{B}}\left(\boldsymbol{\rho}, \boldsymbol{\rho}^{\prime}\right)=\frac{i}{4} \sin ^{2}(\theta) \mathrm{H}_{0}\left(k_{\rho} \varrho\right)+\frac{i}{4} \frac{\cos (2 \theta)}{k_{\mathrm{B}} \varrho} \mathrm{H}_{1}\left(k_{\rho} \varrho\right) \\
G_{x y}^{\mathrm{B}}\left(\boldsymbol{\rho}, \boldsymbol{\rho}^{\prime}\right)=\frac{i}{4} \frac{\sin (2 \theta)}{2} \mathrm{H}_{2}\left(k_{\rho} \varrho\right) \\
G_{y y}^{\mathrm{B}}\left(\boldsymbol{\rho}, \boldsymbol{\rho}^{\prime}\right)=\frac{i}{4} \cos ^{2}(\theta) \mathrm{H}_{0}\left(k_{\rho} \varrho\right)-\frac{i}{4} \frac{\cos (2 \theta)}{k_{\mathrm{B}} \varrho} \mathrm{H}_{1}\left(k_{\rho} \varrho\right)
\end{gathered}
$$$$
G_{z z}^{\mathrm{B}}\left(\boldsymbol{\rho}, \boldsymbol{\rho}^{\prime}\right)=\frac{i}{4} \mathrm{H}_{0}\left(k_{\rho} \varrho\right) .
$$ 
The form of Eq. (36) emphasizes the two independent polarizations that can be excited in that case. For TE polarization the electric field has only two $x, y$ components and $\mathbf{G}^{\mathrm{B}}\left(\boldsymbol{\rho}, \boldsymbol{\rho}^{\prime}\right)$ reduces to a $(2 \times 2)$ matrix; for TM polarization the electric field becomes a scalar (one single $z$ component) and Green's tensor reduces to the scalar Green's function (25) with $z=0$.

Integration of Eq. (9) on a circular mesh centered at $\boldsymbol{\rho}_{i}$ with effective radius $R_{i}^{\text {eff }}$,

$$
R_{i}^{\text {eff }}=\left(\frac{\mathrm{V}_{i}}{\pi}\right)^{1 / 2}
$$

leads to the value of $\mathbf{M}_{i}$ :

$$
\mathbf{M}_{i}=\left(\begin{array}{ccc}
\frac{i \pi}{4} \alpha \gamma & 0 & 0 \\
0 & \frac{i \pi}{4} \alpha \gamma & 0 \\
0 & 0 & \frac{i \pi}{2} \beta \gamma
\end{array}\right) \text {, }
$$

with

$$
\begin{gathered}
\alpha=2-\frac{k_{\rho}^{2}}{k_{\mathrm{B}}^{2}} \\
\beta=1-\frac{k_{z}^{2}}{k_{\mathrm{B}}^{2}} \\
\gamma=\frac{R_{i}^{\mathrm{eff}}}{k_{\rho}} \mathrm{H}_{1}\left(k_{\rho} R_{i}^{\mathrm{eff}}\right)+\frac{2 i}{\pi k_{\rho}^{2}} .
\end{gathered}
$$

When TE and TM polarizations are decoupled, we have in addition $\alpha=\beta=1$.

The corresponding source dyadic for a $2 \mathrm{D}$ system is [12]

$$
\mathbf{L}=\frac{1}{2}\left(\begin{array}{ccc}
1 & 0 & 0 \\
0 & 1 & 0 \\
0 & 0 & 0
\end{array}\right)
$$

Thus we have all the different elements required for investigating $2 \mathrm{D}$ and $3 \mathrm{D}$ scatterers in polarizable backgrounds.

\section{EXAMPLES OF APPLICATIONS}

The aim of this section is to illustrate the application of this approach for 2D and 3D systems. We will not address here the question of the convergence and accuracy of the numerical solution of Eq. (8) but rather refer the interested reader to Ref. [15], where this is discussed in detail. Let us also mention that problems with about 80000 meshes are within the reach of a standard desktop computer using a con-

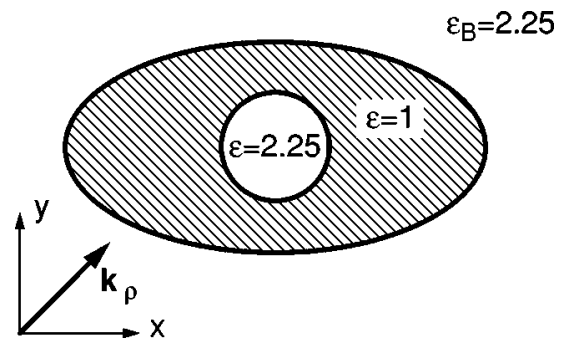

FIG. 2. An elliptical air microcavity in a glass background and with a cylindrical glass core is illuminated by a plane wave propagating in the $\mathbf{k}_{\rho}$ direction. The radius of the cylindrical core is $0.5 \mu \mathrm{m}$ and the ellipse semiaxis are $1 \mu \mathrm{m}$ and $2 \mu \mathrm{m}$. Since both the core and the background have the same permittivity $\varepsilon$, only the air cavity must be discretized (hatched area).

jugate gradients algorithm combined with fast-Fourier transform [13].

\section{A. 2D example: Scattering in a microcavity}

The scattering by $2 \mathrm{D}$ cylindrical structures has always attracted much interest [23-27]. This interest has been augmented recently by the use of cylinders to model the electromagnetic interactions in local probe microscopy [28-30]. Here we would like to use such a configuration to illustrate the coupling between the different field components that can arise when the incident field does not propagate in the $x-y$ plane $\left(\theta_{\mathrm{i}} \neq 90^{\circ}\right.$, Fig. 1$)$.

Let us consider an infinite glass cylinder of permittivity $\varepsilon=2.25$ surrounded by an elliptical air cavity $(\varepsilon=1)$ and embedded in a glass background $\left(\varepsilon_{\mathrm{B}}=2.25\right.$, Fig. 2). Since both the cylinder and the background have the same permittivity, only the air cavity needs to be discretized. Note that the associated dielectric contrast $\Delta \varepsilon(\mathbf{r})$ is then negative, as the cavity has a lower permittivity than its surrounding medium. The wavelength in vacuum for all the simulations presented in this section is $633 \mathrm{~nm}$ and the discretization mesh $67 \times 67 \mathrm{~nm}^{2}$.

We report in Fig. 3(a) the total field intensity for an $s$-polarized field incident at $\theta_{\mathrm{i}}=35^{\circ}$ on the system. For this configuration we observe a very strong scattering at the periphery of the ellipse; on the other hand, almost no field is coupled to the central cylinder. Note also the interference pattern, caused by the interaction of the incoming wave with the reflected one.

For this polarization, the incident field has only $x$ and $y$ components. Nevertheless, the interaction of this incident field with the scattering system leads to an additional $z$ component in the scattered field, as illustrated in Fig. 3(b).

At first sight, the fact that an additional field component can be created during the scattering process, even for a translation symmetrical system, might appear to be quite surprising. Actually, a similar situation occurs when an $s$-polarized plane wave impinges on a tilted interface: the field transmitted through the interface also exhibits an additional component that was not present in the incident field.

These results emphasize that for a 2D system under arbitrary incident illumination, one cannot simply treat TE and TM polarizations separately, but one must carefully take into account the coupling between the different field components, as described in Sec. II C. 

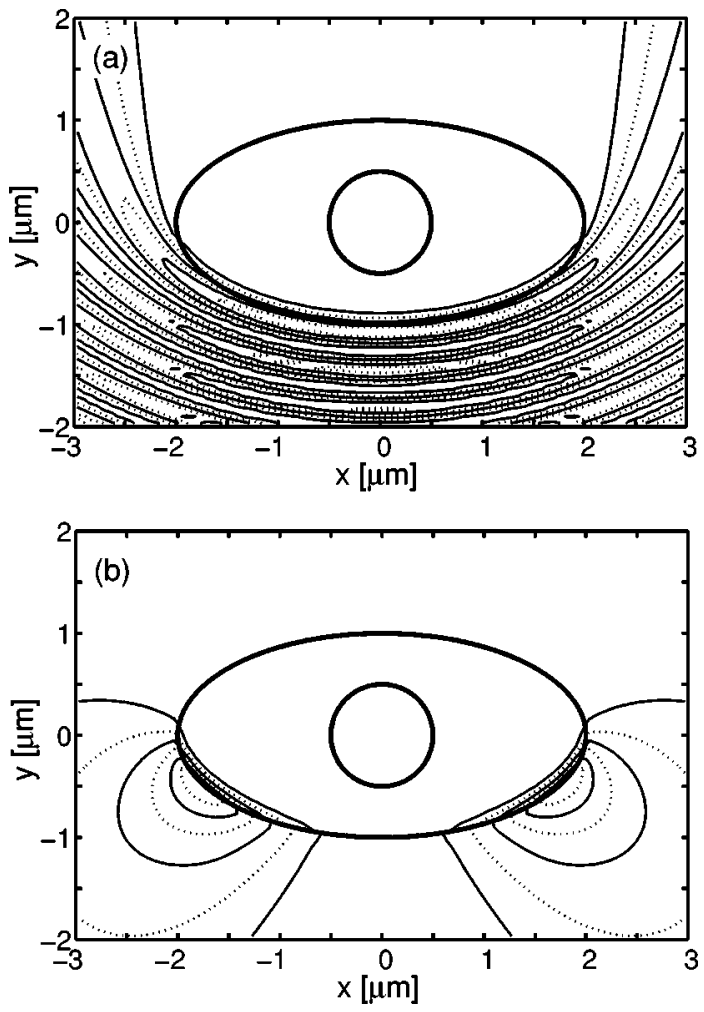

FIG. 3. Field repartition in the microcavity of Fig. 2 when it is illuminated by a $s$-polarized plane wave impinging at an angle $\theta_{i}=35^{\circ}$ propagating parallel to the $y$ axis (Fig. 1); (a) total field intensity and (b) intensity of the $E_{z}$ field component. This last component that is not present in the incident wave is created by the scattering process.

It is interesting to point out that the $E_{z}$ field does not show any interference pattern [Fig. 3(b)]. This again comes from the fact that no such $E_{z}$ component was present in the incident field.

The field repartition in the cylindrical fiber and in the cavity greatly depends on the angle of incidence $\theta_{\mathrm{i}}$ (Fig. 1). For particular incidences, a strong field can be excited in the fiber (Fig. 4). This situation corresponds to the excitation of a mode in the fiber [31]. Such a mode can only exist for particular values of the propagation vector $k_{z}$, i.e., for particular angle of incidences $\theta_{\mathrm{i}}$. In the case of Fig. 4, the mode excited in the fiber reproduces the symmetry of the elliptical cavity.

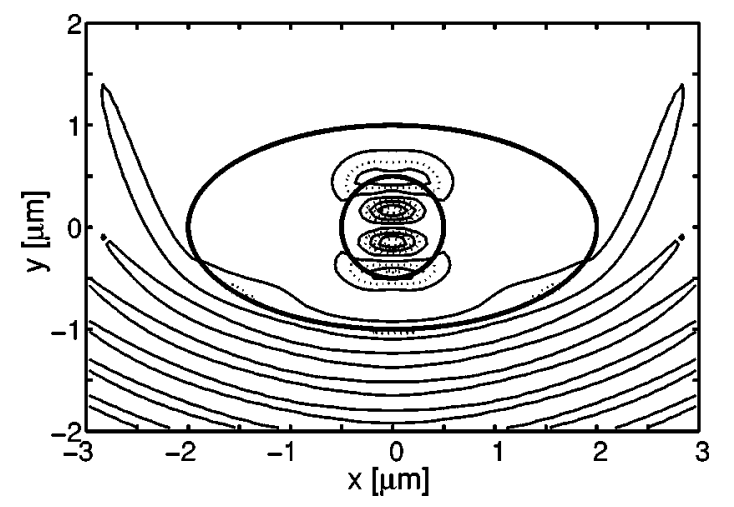

FIG. 4. Same situation as in Fig. 3(a) but for an incident field at $\theta_{\mathrm{i}}=50^{\circ}$. A mode in the cylindrical fiber is now excited in the scattering process.

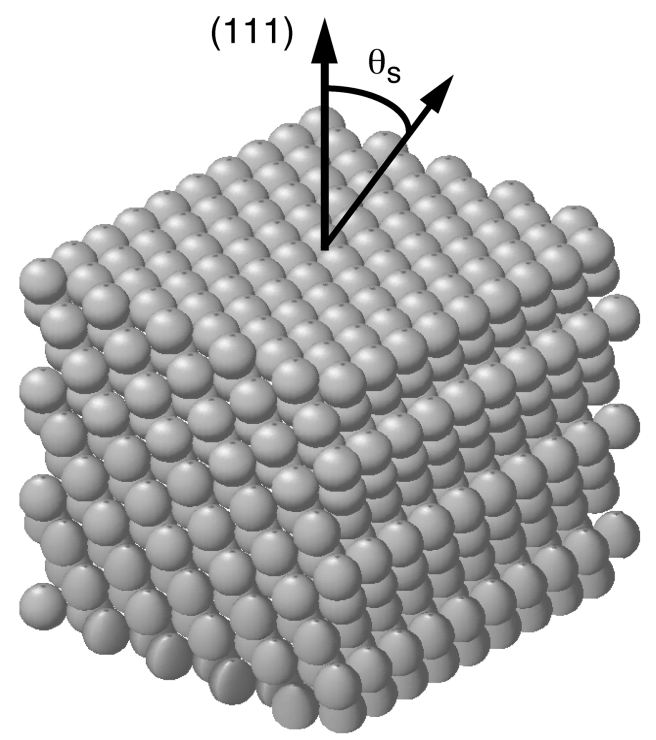

FIG. 5. Model of a cubic close-packed silica spheres domain. The (111) face is illuminated at normal incidence and the field scattered in direction $\theta_{\mathrm{s}}$ is computed.

\section{B. 3D example: color formation in opal}

The flashing colors produced by opal have always been a subject of fascination and it is only in the mid sixties that this colorful behavior could be related to the internal structure of opal. Electron microscopy indeed revealed that this mineraloid is formed by regular arrangements of close-packed silica spheres with a $80-200 \mathrm{~nm}$ radius, in a water background $[32,33]$.

An opal is composed of several small domains containing spheres of a similar radius that are close packed either in cubic (fcc) or hexagonal (hcp) form. Each domain acts as a 3D diffraction grating that scatters light in different directions depending on its wavelength. The interplay of these different domains produces the colorful appearance of opal.

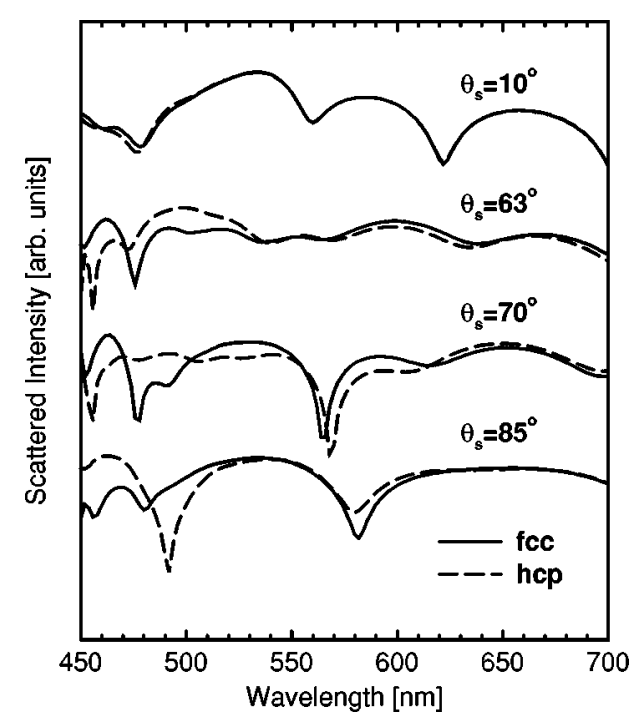

FIG. 6. Scattered intensity (logarithmic scale) as a function of the illuminating wavelength for different scattering directions $\theta_{\mathrm{s}}$ (Fig. 5). Two domains with different crystallographic structures (fcc and hcp) are investigated. 
To illustrate the color formation in opal, let us investigate the scattering properties of such a domain formed by 10 layers of close-packed silica spheres (radius $100 \mathrm{~nm}$ ) in a water background (Fig. 5). The (111) face of the domain is illuminated at normal incidence with a circular polarized plane wave propagating against the face and the field scattered in particular directions $\theta_{s}$ is computed as a function of the wavelength in the visible range $450 \ldots 700 \mathrm{~nm}$. The frequency dependent permittivity for silica and the background are taken from Refs. [34] and [35], respectively. To compute only the scattered field (instead of the total field) at the observation point $\mathbf{r}$ outside the scatterer, one merely sets $\mathbf{E}^{0}(\mathbf{r})=\mathbf{0}$ in Eq. (6) for this observation point.

We compare in Fig. 6 this scattered intensity for an fcc and an hcp domain. For low scattering angles (e.g., $\theta_{s}$ $=10^{\circ}$ ), we see that the spectral behavior does not depend on the crystal structure and both configurations mainly scatter light around $540 \mathrm{~nm}$ in the green. A different behavior is observed for larger scattering angles. For example, at $\theta_{s}$ $=70^{\circ}$, fcc scatters strongly in the blue at $460 \mathrm{~nm}$, whereas hcp does not (Fig. 6). On the other hand, at $\theta_{s}=85^{\circ}$, hcp scatters more in the blue than fcc does.

An opal gem with two such domains would therefore produce distinct color flashes depending on the domain orienta- tion relative to the light source and observer.

\section{CONCLUSION}

As illustrated by the examples of Sec. III, the fully vectorial formalism developed in this paper can be applied to a broad variety of physical problems where scatterers are embedded in a polarizable background. This approach is quite flexible so that it can handle complicated geometries both in 2D and 3D systems. The investigation of 2D configurations has highlighted extremely interesting effects related to the coupling between the different field components. Finally, this formalism can of course also be applied to scattering situations where the scatterers are simply in vacuum $\left(\varepsilon_{\mathrm{B}}\right.$ $=1$ ).

\section{ACKNOWLEDGMENTS}

O.J.F. Martin is most indebted to A. Dereux and C. Girard who introduced him to the power and beauty of the Green's tensor technique. It is also a pleasure to acknowledge stimulating discussions with M. Sundberg, R. Vahldieck, and E. J. Williams. This work was supported by the Swiss National Science Foundation.
[1] P. B. Price and L. Bergström, Appl. Opt. 36, 4181 (1997).

[2] K. Scheerschmidt, R. Hillebrand, and J. Heydenreich, Phys. Status Solidi 116, 123 (1989).

[3] F. K. Hopkins and G. J. Brown, Appl. Opt. 30, 384 (1991).

[4] A. R. Lang, R. Vincent, N. C. Burton, and A. P. Makepeace, J. Appl. Crystallogr. 28, 690 (1995).

[5] K. Takami, Mater. Sci. Eng., B 44, 181 (1997).

[6] B. Chu, Appl. Opt. 36, 7650 (1997).

[7] A. Castiaux, C. Girard, M. Spajer, and S. Davy, Ultramicroscopy 71, 49 (1998).

[8] A. A. Lucas, J.-P. Vigneron, Ph. Lambin, and I. Derycke, Surf. Sci. 269, 74 (1992).

[9] C. Girard, P. Lambin, A. Dereux, and A. A. Lucas, Phys. Rev. B 49, 11425 (1994).

[10] P. Gay-Balmaz, and J. R. Mosig, Int. J. Microwave and Millimeter-wave CAE 3, 330 (1997).

[11] P. M. Morse and H. Feshbach, Methods of Theoretical Physics (McGraw-Hill, New York, 1953), Chap. 13.

[12] A. D. Yaghjian, Proc. IEEE 68, 248 (1980).

[13] B. T. Draine and P. J. Flatau, J. Opt. Soc. Am. A 11, 1491 (1994) and references therein.

[14] W. C. Chew, Waves and Fields in Inhomogeneous Media (IEEE Press, New York, 1995), Sec. 8.9.3.

[15] N. B. Piller and O. J. F. Martin, IEEE Trans. Antennas Propag. (to be published).

[16] P. J. Flatau, Opt. Lett. 22, 1205 (1997).

[17] O. J. F. Martin, C. Girard, and A. Dereux, Phys. Rev. Lett. 74, 526 (1995).

[18] C. Girard, O. J. F. Martin, and A. Dereux, Phys. Rev. Lett. 17, 3098 (1995).

[19] C. Girard and A. Dereux, Phys. Rev. B 49, 11344 (1994).
[20] H. Levine and J. Schwinger, Commun. Pure Appl. Math. 3, 355 (1950).

[21] A. Castaiux, C. Girard, A. Dereux, O. J. F. Martin, and J.-P. Vigneron, Phys. Rev. E 54, 5752 (1996).

[22] I. S. Gradshteyn and I. M. Ryzhik, Table of Integrals Series and Products, 4th ed. (Academic Press, New York, 1965).

[23] J. M. Saiz, P. J. Valle, F. Gonzalez, E. M. Oritz, and F. Moreno, Opt. Lett. 21, 1330 (1996).

[24] C. J. Rodger, J. R. Wait, and R. L. Dowden, Radio Sci. 32, 907 (1997).

[25] T. Rother, K. Schmidt, and J. Wauer, J. Quant. Spectrosc. Radiat. Transf. 57, 669 (1997).

[26] S. P. Savaidis and J. A. Roumeliotis, IEEE Trans. Microwave Theory Tech. 45, 1792 (1997).

[27] P. J. Valle, F. Moreno, and J. M. Saiz, J. Opt. Soc. Am. A 15, 158 (1998).

[28] F. Pincemin, A. Sentenac, and J.-J. Greffet, J. Opt. Soc. Am. A 11, 1117 (1994).

[29] A. Madrazo and M. Nieto-Vesperinas, Appl. Phys. Lett. 70, 31 (1997).

[30] A. Madrazo and M. Nieto-Verperinas, J. Opt. Soc. Am. A 14, 1 (1997).

[31] D. Marcuse, Light Transmission Optics, 2nd ed. (Krieger, Malabar, 1989), Chap. 8.

[32] J. B. Jones, J. V. Sanders, and E. R. Segnit, Nature (London) 204, 990 (1964).

[33] P. J. Darragh and J. V. Sanders, Gems and Gemology 11, 291 (1965).

[34] W. J. Tropf, M. E. Thomas, and T. J. Harris, in Handbook of Optics, 2nd ed., edited by M. Bass (McGraw-Hill, New York, 1995), Vol. II, Chap. 33, Table 22.

[35] C. D. Mobley, in Handbook of Optics, 2nd ed., (Ref. [34], Vol. I, Chap. 43, Table 4. 